Canad. Math. Bull. Vol. 20 (4), 1977

\title{
NONEXPANSIVE MAPPINGS IN LOCALLY CONVEX SPACES
}

BY

TROY L. HICKS AND JOHN D. KUBICEK

Recently Bruck initiated the study of the structure of the fixed-point set of a nonexpansive selfmap $T$ of a Banach space, where $T$ satisfies a conditional fixed point property. We generalize many of his results to a Hausdorff locally convex space $X$. Also, we generalize a result of Holmes and Narayanaswami and use it, along with a procedure of Kiang, to obtain a fixed point theorem for families of asymptotically nonexpansive mappings in $X$.

Let $R^{\Delta}$ be the product of $\Delta$ copies of the real line $R$. We give $R^{\Delta}$ the product topology. Addition and multiplication in $R^{\Delta}$ are defined pointwise. For $f$, $g \in R^{\Delta}$

(1) $f \leq g$ means $f(q) \leq g(q)$ for all $q \in \Delta$;

(2) $f<g$ means $f \leq g$ and there exists $q \in \Delta$ with $f(q)<g(q)$;

(3) $f \ll g$ means $f(q)<g(q)$ for all $q \in \Delta$.

$R^{\Delta}$ is called a Tychonoff semifield [1]. A base for the neighborhood system $U$ of 0 in $R^{\Delta}$ is the collection $\mathscr{B}$ of sets of the form

$$
U\left(\varepsilon ; q_{1}, \ldots, q_{n}\right)=\left\{f:\left|f\left(q_{i}\right)\right|<\varepsilon, \quad \varepsilon>0, \quad q_{i} \in \Delta\right\} .
$$

Throughout this paper $X$ will denote a Hausdorff locally convex space and $C$ a nonempty closed convex subset of $X$. The topology $t$ of $X$ is generated by the family $\left\{N_{q}: q \in \Delta\right\}$ of continuous seminorms. We define $N: X \rightarrow R^{\Delta}$ by $N(x)(q)$ $=N_{q}(x)$. The mapping $\mathrm{N}$ satisfies the axioms of a norm and is called a norm over $R^{\Delta}$. A natural topology $t_{N}$ is induced on $X$ by $N$; a basis of neighborhoods of zero is given by all sets of the form

$$
S(0, U)=\{x: N(x) \in U\},
$$

where $U=U\left(\varepsilon ; q_{1}, \ldots, q_{n}\right) \in \mathscr{B}$. Since $t_{N}=t, X$ is normed over $R^{\Delta}$ by $N$. A metric $\rho$ for $X$ over $R^{\Delta}$ is obtained by defining $\rho(x, y)=N(x-y)$. It is shown in [1] that the mapping $(x, y) \rightarrow \rho(x, y)$ is continuous.

1. Nonexpansive retracts. A mapping $T$ of $X$ into itself is said to be nonexpansive if $N(T x-T y) \leq N(x-y)$ for all $x, y \in X$. The set $\{x: T x=x\}$ of fixed points of $T$ is denoted by $F(T)$. Also, for $A \subset C$ we define $M(A)=$ $\{T \mid T: C \rightarrow C$ is nonexpansive and $A \subset F(T)\}$.

(Received by the editors May 12, 1975 and in revised form, July 8, 1976 and May 3, 1977) 
Lemma 1. Suppose $p$ is a continuous seminorm on $X$ and a net $\left\{x_{d}: d \in D\right\}$ converges weakly to $x \in X$. Then $p(x) \leq \underline{\lim } p\left(x_{d}\right)$.

Proof. There exists a continuous linear functional $f$ such that $f(x)=p(x)$ and $|f(y)| \leq p(y)$ for all $y \in X$. Thus $p(x)=f(x)=\underline{\lim }\left|f\left(x_{d}\right)\right| \leq \lim p\left(x_{d}\right)$.

LemMa 2. Suppose $C$ is weakly compact and $A$ is a non-empty subset of $C$. Then $M(A)$ is compact in the topology of weak pointwise convergence.

Proof. Fix $x_{0} \in A$. For each $x$ define $C_{x}=\left\{y \in C: N\left(y-x_{0}\right) \leq N\left(x-x_{0}\right)\right\}$. For each $T \in M(A)$ and each $x \in C$ we have $T x \in C_{x}$. Thus $M(A) \subset P=$ $\Pi\left\{C_{x}: x \in C\right\}$.

Clearly $C_{x}$ is convex; and since the mapping $x \rightarrow N(x)$ is continuous, $C_{x}$ is closed. Thus each $C_{x}$ is a closed convex subset of the weakly compact set $C$. Therefore $C_{x}$ is weakly compact; and by Tychonoff's theorem, $P$ is compact in the topology of weak pointwise convergence.

To conclude the proof it suffices to show that $M(A)$ is closed in $P$. Suppose $\left\{T_{d}: d \in D\right\}$ is a net in $M(A)$ converging to $T \in P$. Clearly $A \subset F(T)$; and since $T \in P, T(C) \subset C$. Using lemma 1 we have

$$
N_{q}(T x-T y) \leq \underline{\lim } N_{q}\left(T_{d} x-T_{d} y\right) \leq N_{q}(x-y)
$$

for all $x, y \in C$ and $q \in \Delta$. Thus $T$ is nonexpansive and $M(A)$ is closed in $P$.

Lemma 3. Suppose $C$ is weakly compact and $A$ is a nonempty subset of $C$. Then there exists $S \in M(A)$ such that for each $T \in M(A)$ and $x, y \in C$, $N(T S x-T S y)=N(S x-S y)$.

Proof. Define an order on $M(A)$ by $T^{\prime} \leq T$ if $N\left(T^{\prime} x-T^{\prime} y\right) \leq N(T x-T y)$ for all $x, y \in C$. For each $T \in M(A)$ let $I(T)=\left\{T^{\prime} \in M(A): T \leq T\right\}$. Using the same techniques as used in lemma 2 to show $M(A)$ is closed in $P$, we obtain $I(T)$ is closed in $M(A)$. Thus $I(T)$ is weakly compact.

If $\mathscr{C}$ is a chain in $M(A)$, then $\{I(T): T \in \mathscr{C}\}$ is a chain under set inclusion. Since each $I(T)$ is compact there exists $T_{0} \in \bigcap\{I(T): T \in \mathscr{C}\} . T_{0}$ is a lower bound for $\mathscr{C}$. Zorn's lemma now implies that $M(A)$ has a minimal element $S$.

For each $T \in M(A), T S \in M(A)$; and since $T$ is nonexpansive, $N(T S x-$ $T S y) \leq N(S x-S y)$ for all $x, y \in C$. But $S$ is a minimal element in $M(A)$. Thus $N(T S x-T S y)=N(S x-S y)$ for all $x, y \in C$ and $T \in M(A)$.

A subset $A$ of $C$ is a nonexpansive retract of $C$ if either $A=\emptyset$ or there is a retraction of $C$ onto $A$ which is nonexpansive.

A mapping $T: C \rightarrow X$ satisfies the conditional fixed point property (CFPP) if either $T$ has no fixed points, or $T$ has a fixed point in every bounded closed convex subset which it leaves invariant.

With lemmas 2 and 3, the proofs of theorem 1 and 2 are the same as for Banach spaces [2] and hence omitted.

THEOREM 1. Suppose $C$ is weakly compact and $A$ is a nonempty subset of $C$. Suppose for each $z \in C$ there exists $T \in M(A)$ such that $T z \in A$. Then $A$ is a nonexpansive retract of $C$. 
THeOREM 2. Suppose $C$ is weakly compact and $T: C \rightarrow C$ is nonexpansive ard satisfies (CFPP). Then $F(T)$ is a nonexpansive retract of $C$.

The conditional fixed point property does hold for several classes of mappings. Suppose $K$ is a nonempty weakly compact convex subset of $X$ and $K$ has normal structure. Then Tan [8] has shown that a nonexpansive mapping of $K$ into itself has a fixed point. Thus (CFPP) holds for nonexpansive mappings of $C$ into $X$ where $C$ is weakly compact and has normal structure.

A subset $B$ of $X$ is said to have the fixed point property for nonexpansive mappings if every nonexpansive mapping of $B$ into itself has a fixed point. A set $B$ is said to have the hereditary fixed point property (HFPP) if every nonempty bounded closed convex subset of $B$ has the fixed point property for nonexpansive mappings. If $T: C \rightarrow C$ is nonexpansive and $C$ satisfies (HFPP), then $T$ satisfies (CFPP). Thus, if $C$ is bounded and compact and $T: C \rightarrow C$ is nonexpansive, then $T$ satisfies (CFPP).

A sequence $\left\{x_{n}\right\}$ is said to be Cauchy if for each neighborhood $U$ of 0 in $R^{\Delta}$, there exists an integer $M$ such that $N\left(x_{n}-x_{m}\right) \in U$ for all $n, m \geq M$. We say $X$ is sequentially complete if every Cauchy sequence converges.

THeOrem 3. Suppose $X$ is sequentially complete and $T: C \rightarrow C$ is nonexpansive. Then $T$ satisfies (CFPP) if any one of the following hold:

(a) $(I-T)(K)$ is strongly closed whenever $K$ is a bounded closed convex subset of $C$;

(b) $T$ is compact;

(c) $C$ is weakly compact and $T$ is affine.

Proof. Suppose $K$ is a bounded closed convex subset of $C$ with $T(K) \subset K$.

(a) Let $\left\{t_{n}\right\}$ be a sequence from $(0,1)$ such that $\lim t_{n}=1$. By a result of Tan [7, theorem 2.3], the function $t_{n} T$ has a fixed point $x_{n} \in K$. Since $K$ is bounded, $(I-T)\left(x_{n}\right)=\left(1-1 / t_{n}\right)\left(x_{n}\right) \rightarrow 0$. Since $(I-T)(K)$ is closed, there exists $x \in K$ with $T x=x$.

(b) We show $(I-T)(K)$ is closed and the result follows from (a). Suppose $\left\{x_{d}-T\left(x_{d}\right): d \in D\right\}$ is a net in $(I-T)(K)$ converging to $y \in X$. Since $T$ is compact and $\left\{x_{d}\right\}$ is bounded, $\left\{T\left(x_{d}\right)\right\}$ contains a subnet, which we also denote by $T\left(x_{d}\right)$, converging to $y_{1}$. Since $T\left(x_{d}\right) \rightarrow y_{1}$ and $x_{d}-T\left(x_{d}\right) \rightarrow y, x_{d} \rightarrow y_{1}+y=$ $z \in K$. The continuity of $T$ implies $T(z)=y_{1}$. Thus $y=z-y_{1}=z-T z \in$ $(I-T)(K)$.

(c) We again show that $(I-T)(K)$ is closed. Since $T$ is affine, $(I-T)(K)=$ $z_{0}+T^{\prime}(K)$ where $T^{\prime}$ is continuous and linear. Thus it suffices to show that $T^{\prime}(K)$ is closed. Since $K$ is convex and weakly compact, $T^{\prime}(K)$ is also convex and weakly compact. But $X$ is a Hausdorff space, thus $T^{\prime}(K)$ is weakly closed and hence closed.

For families of nonexpansive mappings on $X$ the following results of Bruck [2] carry over, with the same proof, to Hausdorff locally convex spaces. 
TheOREM 4. Suppose $C$ is weakly compact and satisfies (HFPP). Suppose $F$ is a finite family of commuting nonexpansive mappings from $C$ into $C$. Then $\bigcap\{F(T): T \in F\}$ is a nonexpansive retract of $C$.

THeORem 5. Suppose $C$ is compact and $\mathscr{F}$ is an arbitrary family of commuting nonexpansive mappings of $C$ into $C$. Then $\bigcap\{F(T): T \in \mathscr{F}\}$ is a nonempty nonexpansive retract of $C$.

2. Asymptotically Nonexpansive Mappings. A family $\mathscr{F}$ of mappings from $\boldsymbol{X}$ into $X$ is asymptotically nonexpansive [asymptotically isometric] if for each $x, y \in X$ there exists a $S \in \mathscr{F}$ such that for all $T$ in $\mathscr{F}$

$$
\begin{gathered}
N(T S(x)-T S(y)) \leq N(x-y) \\
{[N(T S x-T S y)=N(x-y)]}
\end{gathered}
$$

We recall that the family $\mathscr{B}$ of all sets of the form

$$
U\left(\varepsilon ; q_{1}, \ldots, q_{n}\right)=\left\{f:\left|f\left(q_{i}\right)\right|<\varepsilon, \varepsilon>0, q_{i} \in \Delta\right\}
$$

is a base for $U$, the neighborhood system of 0 in $R^{\Delta}$. For $\mathscr{F}$ a commutative semigroup of continuous asymptotically nonexpansive mappings on $X$ and $Y \subset X$ we define,

(5) $Y^{\mathscr{F}}=\{z \in Y$ : there exists $x \in Y$ such that for each $T \in \mathscr{F}$ and each $U \in \mathcal{U}$, there exists $S \in \mathscr{F}$ with $N(T S(x)-z) \in U\}$.

The following theorem generalizes propositions 1 and 2 of [3].

THEOREM 6. Suppose $\mathscr{F}$ is a commutative semigroup of continuous asymptotically nonexpansive mappings on $X$.

(i) If $z \in X^{\mathscr{F}}$, then for every $T \in \mathscr{F}$ and $U \in \mathcal{U}$, there exists $S \in \mathscr{F}$ with $N(T S(z)-z) \in U$.

(ii) If $z \in X^{\mathscr{F}}$, then $\mathscr{F} \mid \mathscr{F}(z)$ is a family of asymptotic isometries.

(iii) If $A \subset X$ and $\mathscr{F} \mid A$ is a family of asymptotic isometries, then $\mathscr{F} \mid A$ is a family of isometries.

Proof of (i). Suppose $z \in X^{\mathscr{F}}, T \in \mathscr{F}$ and $U \in \mathcal{U}$. There are a finite number of subbase elements $U\left(\varepsilon, q_{i}\right)$ such that $\bigcap_{i} U\left(\varepsilon, q_{i}\right) \subset U$. Let $V=\bigcap_{i} U\left(\varepsilon / 2, q_{i}\right)$, then from (5) there exists $T_{1} \in \mathscr{F}$ with $N\left(T_{1} T(x)-z\right) \in V$. Since $\mathscr{F}$ is commutative and asymptotically nonexpansive, there exists $T_{2} \in \mathscr{F}$ such that $N\left(S T_{2} T_{1} T(x)-S T_{2}(z)\right) \leq N\left(T_{1} T(x)-z\right)$ for all $S \in \mathscr{F}$. Also, from (5), there exists $T_{3} \in \mathscr{F}$ with $N\left(T T_{2} T_{3} T T_{3}(x)-z\right) \in V$. Let $S_{0}=T_{2} T_{3}$. Then, for each $i$, we have

$$
\begin{aligned}
N_{q_{i}}\left(T S_{0}(z)-z\right) & \leq N_{q_{i}}\left(T S_{0}(z)-T T_{2} T_{1} T T_{3}(x)\right)+N_{q_{i}}\left(T T_{2} T_{1} T T_{3}(x)-z\right) \\
& \leq N_{1_{i}}\left(z-T_{1} T(x)\right)+\varepsilon / 2<\varepsilon
\end{aligned}
$$

Therefore, $N\left(T S_{0}(z)-z\right) \varepsilon U$. 
Proof of (ii). Suppose it is not true. Then there exist $u=T_{1}(z)$ and $v=T_{2}(z)$ such that for each $T \in \mathscr{F}$ there exists $S \in \mathscr{F}_{F}$ and $q \in \Delta$ with $\left.N_{q}(T S u-T S v)\right) \neq N_{q}(u-v)$. Since $\mathscr{F}$ is asymptotically nonexpansive, there exists $T_{3} \in \mathscr{F}$ such that $N\left(S T_{3}(u)-S T_{3}(v)\right) \leq N(u-v)$ for all $S \in \mathscr{F}$. Thus there exists $S_{0} \in \mathscr{F}$ and $q \in \Delta$ such $N_{q}\left(S_{0} T_{3}(u)-S_{0} T_{3}(v)\right)<N_{q}(u-v)$. Let $\varepsilon=$ $-N_{q}\left(S_{0} T_{3}(u)+S_{0} T_{3}(v)\right)+N_{q}(u-v)$ and $V=U(\varepsilon / 2, q)$. By (4), there exists $T_{4} \in \mathscr{F}$ such that

$$
N\left(S T_{4} S_{0} T_{3}(u)-S T_{4} S_{0} T_{3}(v)\right) \leq N\left(S_{0} T_{3}(u)-S_{0} T_{3}(v)\right)
$$

for all $S \in \mathscr{F}$.

Since $T_{1}$ and $T_{2}$ are continuous at $z$, there exists a $U \in \mathcal{U}$ such that $N(y-z) \in U$ implies $N\left(T_{1}(y)-T_{1}(z)\right)$ and $N\left(T_{2}(y)-T_{2}(z)\right)$ are in $V$. Applying (i) to $T_{4} S_{0} T_{3}=S_{1}$ and $U$, there exists $T_{5} \in \mathscr{F}$ such that $N\left(T_{5} S_{1}(z)-z\right) \in U$. Since $u=T_{1}(z), v=T_{2}(z)$ and $N\left(T_{5} S_{1}(z)-z\right) \in U$, we have $N\left(T_{5} S_{1}(u)-u\right) \in V$ and $N\left(T_{5} S_{1}(v)-v\right) \in V$. Finally using (6) we obtain

$$
\begin{aligned}
N_{q}(u-v) & \leq N_{q}\left(u-T_{5} S_{1}(u)\right)+N_{q}\left(T_{5} S_{1}(u)-T_{5} S_{1}(v)\right) \\
& +N_{q}\left(T_{5} S_{1}(v)-v\right) \\
& <\varepsilon / 2+N_{q}\left(S_{0} T_{3}(u)-S_{0} T_{3}(v)+\varepsilon / 2=N_{q}(u-v) .\right.
\end{aligned}
$$

This contradiction implies $\mathscr{F} \mid \mathscr{F}(z)$ is a family of asymptotic isometries.

Proof of (iii). Suppose $x, y \in A$ and $T \in \mathscr{F}$. Then there exist $T_{1}, T_{2} \in \mathscr{F}$ such that $N\left(S T_{1}(x)-S T_{1}(y)\right)=N(x-y)$ and $N\left(S T_{2} T(x)-S T_{2} T(y)\right)=N\left(T_{x}-T y\right)$ for all $S \in \mathscr{F}$. Since $T_{1}$ and $T_{2} T$ are in $\mathscr{F}$, we have

$$
\begin{aligned}
N(x-y) & =N\left(T_{2} T T_{1}(x)-T_{2} T T_{1}(y)\right) \\
& =N\left(T_{1} T_{2} T x-T_{1} T_{2} T y\right)=N(T x-T y) .
\end{aligned}
$$

REMARK. The proof of theorem 6 does not use the norm property that $N(a x)=a N(x)$. Thus if $X$ is a Hausdorff uniform space, hence metrizable over $R^{\Delta}$, the theorem is valid with the same proof.

We say a subset $K$ of $X$ if strictly convex if for $x, y \in K, N(x+y)=$ $N(x)+N(y)$ implies $\{x, y\}$ is linearly dependent.

Suppose $\mathscr{F}$ is a commutative semigroup of continuous asymptotically nonexpansive mappings on $X$ and $z \in X^{\mathscr{F}}$. Then by theorem 6(i), for each $T \in \mathscr{F}$ and each $U \in \mathcal{U}$, there exists $\varphi_{T, U} \in \mathscr{F}$ such that $N\left(T \varphi_{T, U}(z)-z\right) \in U$. Let $B=$ $\left\{x \in X: N\left(x-\Phi_{T, V} T(z)\right) \in V\right.$ for all $T \in \mathscr{F}$ and for all $\left.V \in \mathscr{B}\right\}$ where $\mathscr{B}$ is the base for $U$ define in the introduction. $B$ is not empty since $z \in B$. The following theorem generalizes theorem 5 of Kiang [5].

THEOREM 7. Suppose $C$ is a weakly compact subset of a strictly convex space $X$ and $\mathrm{F}: C \rightarrow C$ is a cummutative semigroup of continuous asymptotically nonex- 
pansive mappings on $C$. Suppose $z \in X^{\mathscr{F}} \neq \emptyset$. Then $\mathscr{F}$ has a common fixed point in the closed convex hull of $\{z\} \cup \mathscr{F}(z)$.

The proof of theorem 7 is based on the following lemmas concerning the set $\mathscr{B}_{B}$ defined in the preceeding paragraph.

LEMMA 4. $T(B) \subset B$ for all $T \in \mathscr{F}$.

Proof. Let $T \in \mathscr{F}$. If $x \in B$, then $x \in X^{\mathscr{F}}$. Thus by theorem $6, \mathscr{F} \mid \mathscr{F}(x)$ is a family of isometries. Therefore, for $T \in \mathscr{F}, \quad N\left(T(x)-\Phi_{S, V} S T(x)\right)=$ $N\left(x-\Phi_{S, V} S(x)\right) \in V$ for all $V \in \mathscr{B}$ and all $S \in \mathscr{F}$. Thus $T x \in B$.

LEMMA 5. $\mathscr{F} \mid \bar{B}$ is a family of isometries.

The proof of lemma 5 is the same as Kiang's proof of iemma $2[5$, p. 68] and hence is omitted.

Lemma 6. Suppose $X$ is strictly convex, then for every $T \in \mathscr{F}, x, y \in B$ and $\lambda \in$ $[0,1] ; T\left(\lambda x_{1}+(1-\lambda) x_{2}\right)=\lambda T\left(x_{1}\right)+(1-\lambda) T\left(x_{2}\right)$.

Proof. Partially order $\mathscr{B}$ by $V_{1} \geq V_{2}$ if $V_{1} \subset V_{2}$. Then, for $x \in B$ and $T \in \mathscr{F}$, the net $\left\{\Phi_{T, V} T(x): V \in B\right\}$ converges to $x$. Using this, the Banach space proof of Kiang [5] carries over to locally convex spaces and hence the details are omitted.

L.emma 7. Suppose $X$ is sirictly convex. Then $\bar{B}$ is convex.

Proof. We show that $B$ is convex. Let $x_{1}, x_{2} \in B, \lambda \in[0,1]$ and $x=$ $\lambda x_{1}+(1-\lambda) x_{2}$. Then by definition of $B$, for $i=1,2, N\left(x_{i}-\Phi_{T, V} T x_{i}\right) \in V$ for all $T \in \mathscr{F}$ and all $V \in \mathscr{B}$. Let $T \in \mathscr{F}$ and $V \in \mathscr{B}$. Since $V \supset \bigcap_{j=1}^{n} U\left(\varepsilon, q_{j}\right)$, using lemma $\delta$, we have

$$
\begin{aligned}
N_{q_{j}}\left(x-\Phi_{T, V} T x\right)= & N_{q_{i}}\left[\lambda\left(x_{1}-\Phi_{T, V} T x_{1}\right)+(1-\lambda)\left(x_{2}-\Phi_{T, V} T x_{2}\right)\right] \\
& \leq \lambda N_{q_{j}}\left(x_{1}-\Phi_{T, V} T x_{1}\right)+(1-\lambda) N_{q_{j}}\left(x_{2}-\Phi_{T, V} T x_{2}\right) \\
& <\varepsilon \text { for each } j .
\end{aligned}
$$

Thus $N\left(x-\Phi_{T, V} T x\right) \in V$. Therefore, $B$ and hence $\bar{B}$ are convex.

Lemma 8. Suppose $B$ is a closed convex subset of a $T_{2}$ locally convex space $X$ and $T: B \rightarrow B$ is contiruous and affine. Then $T$ is weakly continuous.

Proof. Suppose $T$ is not weakly continuous. Then there exists a net $\left\{x_{d}\right\}$ in $B$ that converges weakly to $x$ in $B$ and a continuous linear functional $f$ such that $f\left(T x_{d}\right) \nrightarrow f(T x)$. Hence there exists an $\varepsilon>0$ and a subnet $\left\{x_{\alpha}\right\}$ of $\left\{x_{d}\right\}$ such that for each $\alpha,\left|f\left(T x_{\alpha}\right)-f(T x)\right| \geq \varepsilon$. Without loss of generality we may assume that $f\left(T x_{\alpha}\right)-f(T x) \geq \varepsilon$ for all $\alpha$. Since $\left\{x_{\alpha}\right\}$ converges weakly to $x$, there exists a net $\left\{y_{\beta}\right\}$ that converges strongly to $x$ where each $y_{\beta}$ is a convex combination of the 
$x_{\alpha}$ 's. Now since $\sum_{i=1}^{n} \alpha_{i}=1, f$ is linear and $T$ is affine, we have

$$
\begin{aligned}
f\left(T y_{\beta}\right)-f(T x) & =f\left[\left(\sum_{i=1}^{n} \alpha_{i} x_{\alpha_{i}}\right)\right]-f(T x) \\
& =f\left(\sum_{i=1}^{n} \alpha_{i} T x_{\alpha_{i}}\right)-f(T x) \\
& =\sum_{i=1}^{n} \alpha_{i} f\left(T x_{\alpha_{i}}\right)-f(T x) \\
& =\sum_{i=1}^{n} \alpha_{i}\left[f\left(T x_{\alpha_{i}}\right)-f(T x)\right] \\
& \geq \sum_{i=1}^{n} \alpha_{i} \varepsilon=\varepsilon
\end{aligned}
$$

However $y_{\beta} \rightarrow x$ and $f$ and $T$ are continuous gives the contradiction that $f\left(T y_{\beta}\right)-f(T x) \rightarrow 0$.

Proof of Theorem 7. Lemmas 4-8 imply that $\mathscr{F} \mid \bar{B}$ is a commutative semigroup of weakly continuous affine isometries and the remainder of the proof is the same as for Banach spaces [5] and hence is omitted.

Examples of a semigroup of continuous asymptotically non-expansive mappings which are not nonexpansive and a non-normable locally convex space which is strictly convex can be constructed. For these and other examples see [10].

\section{REFERENCES}

1. M. Y. Antonovskii, V. G. Boltyanskii and T. A. Sarymsakov, An outline of the theory of topological semi-fields, Russian Math. Surveys, 21 (1966), 163-192.

2. R. E. Bruck, Properties of fixed point sets of nonexpansive mappings in Banach spaces, Trans. Amer. Math. Soc., 179 (1973), 251-262.

3. R. D. Holmes and P. P. Narayanaswami, On asymptotically nonexpansive semigroups of mappings, Can. Math. Bull., 13 (1970), 209-214.

4. J. Horvath, Topological Vector Spaces and Distributions, Addison-Wesley, Reading, Mass., 1966.

5. M. T. Kiang, Fixed point theorems for certain classes of semigroups of mappings, Trans. Amer. Math. Soc., 189 (1974), 63-76.

6. K. K. Tan, Cornmon fixcd point theorems for almost weakly periodic nonexpansive mappings, Proc. Amer. Math. Soc.. 33 (1972), 355-360.

7. - Fixed point theorems for nonexpansive mappings, Pacific Math. J., 41 (1972), 829-842.

8. - Some fixed point theorems for nonexpansive mappings in Hausdorf locally convex spaces, Ph.D. Thesis, University of British Columbia, 1970.

9. A. E. Taylor, Introduction to Functional Analysis, Wiley, New York, 1958.

10. John D. Kubicek, Applications of Metrics over Tikonov semifields and fixed points in Hilbert spaces, Ph.D. Thesis, University of Missouri-Rolla, 1975.

Defartment of Mathematics

UNIVERSITY OF MISSOURI-ROLLA

ROLlA, Missouri 65401 